\title{
PRODUÇÃO DE MICÉLIO EXTERNO POR FUNGOS MICORRÍZICOS ARBUSCULARES E CRESCIMENTO DA SOJ A EM FUNÇÃO DE DOSES DE FÓSFORO(1)
}

\author{
M. A. NOGUEIRA(2) \& E.J . B. N. CARDOSO(3)
}

\begin{abstract}
RESUMO
E m uma associação entre duas espécies de fungos micorrízicos arbusculares (FMAs) (Glomus intraradices ou Gigaspora margarita) e soja (Glycine max L. Merrill cv. IAC-8), avaliaram-se a produção de massa de material seco da parte aérea (MSPA), a massa de material seco de vagens (MSV), a colonização radicular, a concentração de $\mathrm{N}, \mathrm{P}, \mathrm{K}, \mathrm{Ca}, \mathrm{Mg}, \mathrm{S}, \mathrm{Cu}, \mathrm{Fe}, \mathrm{Mn}$ e $\mathrm{Zn}$ na parte aérea e vagens, além das estimativas do comprimento de micélio externo ativo (MEA) e micélio externo total (MET). As plantas receberam cinco doses de fósforo $(0,25,50,100$ e $200 \mathrm{mg} \mathrm{kg}^{-1}$ ) e as avaliações foram feitas em quatro épocas (15, 30, 60 e 90 dias). A inoculação da soja com G. margarita resultou em redução transitória de crescimento aos 60 dias, enquanto aumentos na MSPA devidos à inoculação com os F MAs foram mais evidentes aos 90 dias. A colonização radicular, a produção de MEA e de MET apresentaram relação negativa com o incremento das doses de $P$ nos tratamentos com FMAs. A menor produção de MEA e a fase de crescimento lento mais prolongada observadas no tratamento com G. margarita podem ter colaborado para a redução do crescimento das plantas aos 60 dias. 0 comprimento de MEA produzido por G. intraradices aumentou com o tempo e diminuiu com o aumento das doses de P. Esses efeitos foram menos evidentes para G. margarita. As concentrações de Fe e Mn nas vagens aos $\mathbf{9 0}$ dias foram menores nos tratamentos inoculados com FMAs nas menores doses de $P$, nas quais foi observada maior colonização radicular.
\end{abstract}

Termos para indexação: Micorriza arbuscular, micélio externo, ferro, manganês.

(1) Parte da Tese de Mestrado do primeiro autor, apresentada à Escola Superior de Agricultura "Luiz de Queiroz" em outubro de 1997. Parcialmente apresentado no XXVI Congresso Brasileiro de Ciência do Solo, Rio deJ aneiro, 1997. Recebido para publicação em outubro de 1997 e aprovado em dezembro de 1999.

(2) Pós-Graduando em Solos e Nutrição de Plantas, Escola Superior de Agricultura Luiz de Queiroz - ESALQ. Caixa Postal 9, CEP 13418-900. Piracicaba (SP). Bolsista do CNPq. E-mail: nogueira@carpa.ciagri.usp.br

(3) Professora Titular do Departamento de Solos e Nutrição de Plantas, ESALQ/USP. Bolsista do CNPq. E-mail: ejbncard@carpa.ciagri.usp.br. 


\title{
SUMMARY: EXTERNAL MYCELIUM PRODUCTION BY ARBUSCULAR MYCORRHIZAL FUNGI AND GROWTH OF SOYBEAN FERTILIZED WITH PHOSPHORUS
}

\begin{abstract}
The objective of this work was to study the association between soybean (Glycine max L. Merr. Cv. IAC 8) and two different arbuscular-mycorrhizal (AM) fungi (Gigaspora margarita or Glomus intraradices), grown under increasing rates of phosphorus $(0,25,50,100$ and $200 \mathrm{mg} \mathrm{kg}^{-1}$ ) and harvested at four times (15, 30, 60 and 90 days). The assessed variables were: shoot dry weight, pod dry weight, root col onization by AM fungi, theconcentrations of $\mathrm{N}, \mathrm{P}, \mathrm{K}, \mathrm{S}, \mathrm{Ca}, \mathrm{Mg}, \mathrm{Fe}, \mathrm{Cu}, \mathrm{Mn}$, and $\mathrm{Zn}$ in shoots and pods, estimation of thelength of total external mycelium (TEM) and the length of active external mycelium (AEM). There was a temporal growth reduction in the soybean-G. margarita association at 60 days, and shoot dry weight increasedueto AM fungus col onization was moreevident at 90 days. Increasing $P$ rates decreased root col onization in both fungi, likewisewith TEM and AE M. Col onization by G. margarita was less efficient in a soybean symbiosis because it grew slower than G. intraradices and presented less AEM. This may have been the reason for growth reduction in the soybean-G. margarita association at 60 days. Length of AEM in G. intraradices inoculated soils increased with time and decreased with increasing $P$ rates. Changes in AE M wereless evident for thesoybean-G. margarita. I ron and manganeseconcentrations in pods at 90 days werelower in plants col onized by AM fungi at low $\mathrm{P}$ rates, wherecolonization levels werehigh.
\end{abstract}

Index terms: Arbuscular mycorrhiza, external mycelium, iron, manganese

\section{INTRODUÇÃO}

A colonização radicular ea resposta do hospedeiro à inoculação com fungos micorrízicos arbusculares (FMAs) dependem da interação do sistema simbionte e as mais variadas condições, tais como: as características do sol o (Pacovsky et al., 1986; Sylvia, 1988; Lambais \& Cardoso, 1990) e a fase de desenvol vimento do hospedeiro (Bethlenfal vay et al . 1982d). A disponibilidade de $P$ é o fator edáfico que mais afeta as mi corrizas arbusculares, havendo uma relação inversa com a dependência mi corrízica (Ojala et al., 1983; Cardoso et al., 1986).

Segundo Abbott \& Robson (1981), a falta de relação entre infectividade e eficiência do FMA em promover o crescimento de culturas anuais pode estar relacionada com o tempo necessário ao estabel ecimento da colonização radicular. Lambais $\&$ Cardoso (1990) observaram que o processo de colonização radicular deStyl osanthes guianensis por Gigaspora margarita foi mais lento do que o observado para outras espécies de FMAs, o que, provavelmente, limitou a resposta da planta à micorrização por essa espécie de FMA.

Em alguns casos, não raramente observa-se que a inoculação de plantas com FMAs pode resultar na redução do crescimento do hospedeiro nos estádios iniciais, quando há grande deslocamento de fotoassimilados para o endófito (Bethlenfalvay et al., 1982b).
Alguns autores têm vol tado suas atenções para o estudo da relação entre o micél io interno eo externo à raiz (Bethlenfal vay et al. 1982a; Abbott \& Robson, 1985) e seu impacto no crescimento do hospedeiro. O crescimento da planta pode variar com a proporção entre micélio fúngico interno e externo (Abbott \& Robson, 1981). Respostas negativas (parasitismo) podem ser resultantes de uma grande colonização radicular pelo FMA e pouco crescimento externo (Bethlenfalvay et al. (1982c). De forma contrária, o parasitismo também poderá ocorrer quando houver pouca colonização radicular e grande produção de micélio externo (Cardoso-Filho et al., 1999).

Os fatores que influenciam a colonização micorrízica, como a disponibilidade de $\mathrm{P}$, também atuam na porção externa da simbiose (Nagahashi et al., 1996). Alguns autores têm observado correlação positiva entre comprimento de micélio externo total (MET) e disponibilidade de $P$ no substrato (Cardoso-Filho et al., 1999; Melloni \& Cardoso, 1999b). Todavia, esses resultados contrariam as observações de Abbot \& Robson (1985) e Miranda \& Harris (1994), os quais observaram decréscimos nas quantidades de MET com o incremento do teor de $\mathrm{P}$ no solo.

O micélio externo ativo (MEA) tem si do avaliado pelo uso de diacetato de fluoresceína (FDA) (Schubert et al., 1987; Hamel et al., 1990). Melloni \& Cardoso (1999b) verificaram que o comprimento de MEA variou com o hospedeiro e com a espécie de 
fungo micorrízico, além de apresentar correlação negativa com o aumento da disponibilidade de P no substrato.

O objetivo deste trabal ho foi avaliar, em quatro épocas do ciclo da cultura, a infectividade e a eficiência de duas espécies de FMAs em soja em resposta a doses crescentes de $P$, a produção de MEA e MET, bem como o efeito da micorrização sobre a concentração de N, P, K, S, Ca, Mg, Cu, Fe, Mn eZn na parte aér ea e vagens.

\section{MATE RIAL E MÉTODOS}

O substrato de cultivo foi obtido pela mistura de uma amostra de solo classificado como Areia Quartzosa, série paredão vermel ho distrófico, retirado da camada de $0-20 \mathrm{~cm}$, no município de Piracicaba e areia de rio (3:1, v/v), lavada por cinco vezes. O uso desse substrato teve a finalidade de facilitar a remoção das raízes e do mi célio externo.

Após a autoclavagem do substrato para a eliminação dos FMAs nativos, sua análise química revel ou os seguintes resultados: $\mathrm{pH} \mathrm{CaCl}_{2} 4,0 ;$ M.O.

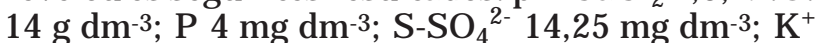
0,4; $\mathrm{Ca}^{2}+5 ; \mathrm{Mg}^{2+} 2 ; \mathrm{Al}^{3}+11 ; \mathrm{H}+\mathrm{Al} 31 ; \mathrm{S} \mathrm{7}, \mathrm{T}$ $38 \mathrm{mmol}_{\mathrm{c}} \mathrm{dm}^{-3}$; Cu 0,42; Fe93; Mn 11; Zn 1,62 $\mathrm{mg} \mathrm{kg}^{-1}$. Com base nesses resultados, foi calculada a necessidade de calagem pelo método da saturação por bases, visando sua el evação a $80 \%$ e a adubação com K e S (Raij et al., 1996). Foram adicionados $0,67 \mathrm{~g} \mathrm{~kg}^{-1}$ de calcário dolomítico, PRNT 131\%, incubando-se por 15 dias. Após esse período, adicionaram-se $0,04 \mathrm{~g} \mathrm{~kg}^{-1}$ de $\mathrm{KCl}\left(50 \% \mathrm{~K}_{2} \mathrm{O}\right)$ e $0,04 \mathrm{~g} \mathrm{~kg}^{-1}$ de gesso $\left(\mathrm{Ca}_{2} \mathrm{SO}_{4} \cdot 2 \mathrm{H}_{2} \mathrm{O}, 18 \% \mathrm{~S}\right)$. Não foi fornecido $\mathrm{N}$ mineral.

O delineamento experimental foi inteiramente casualizado, em esquema fatorial ( $3 \times 5 \times 4)$, correspondente a três tratamentos fúngicos (controle, Glomus intraradices ou Gigaspora margarita), cinco doses de $P(0,25,50,100$ e $200 \mathrm{mg} \mathrm{kg}^{-1}$ ) na forma de superfosfato triplo moído $(<0,5 \mathrm{~mm})$ e quatro épocas de avaliação $(15,30,60$ e 90 dias após o transplantio), com três repetições.

Cada unidade experimental foi constituída de um vaso de cerâmica de $4 \mathrm{dm}^{3}$, impermeabilizado e desinfestado internamente, antes de receber $4 \mathrm{~kg}$ do substrato $\left(3 \mathrm{dm}^{3}\right)$. As sementes foram prégerminadas em areia por três dias. Transplantaramse cinco plântulas do cultivar IAC-8 por parcela, inoculadas no momento do transplantio com as estirpes Semia 587 e Semia 5019 de Bradyrhizobium japonicum. Após uma semana, foi feito o desbaste, mantendo-se duas plantas, ocasião em que receberam, na forma de solução, os seguintes micronutrientes, em $\mathrm{mg} \mathrm{kg}^{-1}$ de substrato: $\mathrm{B}-0,25$ $\left(\mathrm{H}_{3} \mathrm{BO}_{3}\right) ; \mathrm{Cu}-0,75\left(\mathrm{CuSO}_{4} \cdot 5 \mathrm{H}_{2} \mathrm{O}\right) ; \mathrm{Mo}-0,05$ $\left(\mathrm{Na}_{2} \mathrm{MoO}_{4} \cdot 2 \mathrm{H}_{2} \mathrm{O}\right)$ e $\mathrm{Zn}-2,5\left(\mathrm{ZnSO}_{4} \cdot 7 \mathrm{H}_{2} \mathrm{O}\right)$.
A infestação do substrato com cada F MA foi feita com uma suspensão de esporos extraídos por peneiramentoúmido (Gerdemann \& Nicolson, 1963), provenientes devasos de multiplicação em Brachiaria decumbens, seguido de centrifugação em sol ução de sacarose $500 \mathrm{~g} \mathrm{~L}^{-1}$. O volume de cada suspensão foi ajustado para possibilitar a obtenção de cerca de 200 esporos ao pipetar $10 \mathrm{~mL}$, os quais foram adicionados e incorporados nos cinco centímetros superficiais do substrato. O tratamento-controle (sem FMA) recebeu o mesmo volume de suspensão proveniente de vasos com Brachiaria sem FMAs, visando manter a mesma mi crobiota dos tratamentos que receberam FMAs. O experimento foi realizado em casa de vegetação de agosto a novembro de 1996, tendo as plantas recebido irrigações diárias comágua destilada, conforme a necessidade.

Em cada época de colheita, as plantas foram lavadas, secas em estufa a $65^{\circ} \mathrm{C}$, determinando-se a massa do material seco da parte aérea (MSPA = fol has + pecíolos + ramos + vagens). As vagens também tiveram sua matéria seca determinada separadamente (MSV). As vagens e a parte aérea remanescente (fol has + pecíolos + ramos $=$ parte vegetativa) foram moídas separadamente e submetidas a determinações dos teores de $\mathrm{N}$ por destilação semi-microKjeldahl após digestão sulfúrica. Determinaram-setambém $\mathrm{P}$ (colorimetria), $\mathrm{S}$ (turbidimetria), $\mathrm{K}, \mathrm{Ca}, \mathrm{Mg}, \mathrm{Cu}, \mathrm{Fe}, \mathrm{Mn}$ e $\mathrm{Zn}$ (espectrofotometria de absorção atômica) após digestão nítrico-perclórica (Sarruge \& Haag, 1974). As raízes foram retiradas do substrato, lavadas e armazenadas em AFA ( 2 L de ál cool etílico; $0,5 \mathrm{~L}$ de formol; $0,1 \mathrm{~L}$ deácido acético glacial em $4 \mathrm{~L}$ de água destilada) até o momento da determinação da percentagem de colonização radicular pelo método de interseção em placa quadriculada (Giovanetti \& Mosse, 1980) após a col oração das raízes (Phillips \& Hayman, 1970). A extração do micélio externo foi feita pelo método Cardoso-Filho et al. (1999), modificado por Melloni \& Cardoso (1999a), colocandose a membrana com os micélios sobre uma lâmina de microscópio e não entre duas lâminas, conforme método original. A estimativa do comprimento do micélio externo ativo e total foi feita segundo Schubert et al . (1987). Os resultados foram submetidos à análise de variância, desdobrando-se os fatores, ou a interação entre eles, com aplicação do teste t de Student aos fatores qual itativos eanálises de regressão aos fatores quantitativos. Para a estabilização da variância, os dados de percentagem de col onização radicular foram transformados em arc sen $(x / 100)^{1 / 2}$.

\section{RESULTADOS E DISCUSSÃO}

\section{Colonização Radicular}

As raízes das plantas não inoculadas com FMAs foram analisadas e apresentaram-se isentas de 
colonização em todas as épocas. Aos 15 dias, também não havia colonização radicular, mesmo nos tratamentos inoculados com FMAs. Bethlenfalvay et al. (1982b) também não observaram colonização aos 14 dias em plantas desoja inoculadas com F MAs.

A primeira constatação de col onização radicular foi feita aos 30 dias (Figura 1). Nessa época, a percentagem de colonização para ambos FMAs foi menor que 5\%, não havendo diferenças estatísticas entre os tratamentos inoculados em qual quer dose de P. À exceção dos tratamentos com 100 e 200 mg kg-1 $^{-1}$ de $P$, os quais limitaram a evol ução da colonização radicular, a fase exponencial da colonização por $\mathrm{G}$. intraradices ocorreu entre 30 e 60 dias, enquanto G. margarita apresentou pouca evolução nesse interval o. G. intraradices atingiu $75 \%$ de col onização aos 60 dias no tratamento que não recebeu $P$, havendo diminuição da col onização com o aumento das doses de P. Minhoni et al. (1993), estudando a simbiose de Glomus macrocarpum em soja, observaram apenas uma tendência de diminuição da colonização radicular com o aumento das doses de $\mathrm{P}$ em que a máxima foi de $70 \mathrm{mg} \mathrm{kg}^{-1}$. Faquin (1988) também observou quea col onização radicular não foi influenciada mesmo na dose de $90 \mathrm{mg} \mathrm{kg}^{-1}$ de $P$. Contudo, deve-se salientar que naqueles trabalhos foram utilizados sol os oxídicos, nos quais a disponibilidade de $\mathrm{P}$ certamente foi restringida pela fixação do elemento, tornando-o menos disponível. Siqueira et al. (1984), utilizando solo arenoso, também observaram relação inversa entre col onização radicular de soja e disponi bilidade deP, a qual caiu de $60 \%$, na dose 0 , para $10 \%$, na dose $160 \mathrm{mg} \mathrm{kg}^{-1}$, aos 70 dias.

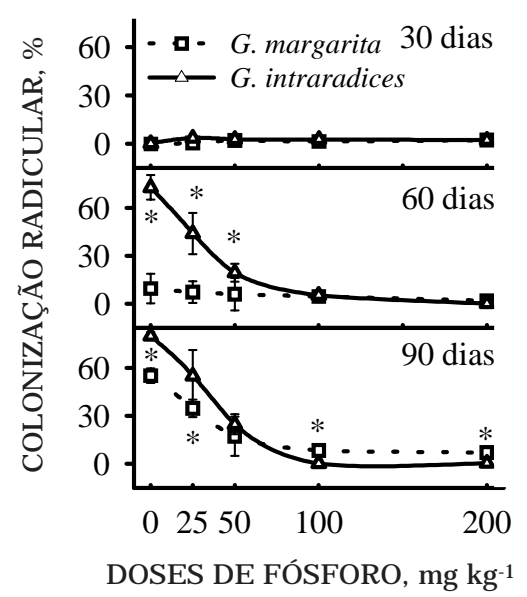

Figura 1. Colonização radicular de soja por fungos micorrízicos arbusculares, considerando as doses de $P$, aos 30, 60 e 90 dias do transplantio. * Diferenças significativas entre fungos na mesma dose, pelo teste t a $5 \%$. Barras verticais representam o desvio-padrão de três repetições, quando maiores que os símbolos.
G. margarita apresentou fase exponencial de colonização radicular apenas entre 60 e 90 dias, especialmente no tratamento sem adição de $P$ e na dose $25 \mathrm{mg} \mathrm{kg}^{-1}$. Fase de crescimento lento mais prolongada de G. margarita também foi observada por Lambais \& Cardoso (1990). Diferentes espécies ou populações de FMAs apresentaram curvas variadas de colonização de acordo com o tempo, dependendo das condições edáficas (Abbott \& Robson, 1985). A interação genética do fungo com a planta talvez seja o fator de maior importância no que se refere à col onização pel os F MAs (Lambais \& Cardoso, 1990). Aos 90 dias e nas doses de P de 100 e $200 \mathrm{mg} \mathrm{kg}^{-1}$, G. margarita apresentou col onização radicular significativamente maior (cerca de 15\%) em relação a G. intraradices (cerca de 3\%). Cardoso et al. (1986) observaram que a diminuição da micorrização de citrus, decorrente do aumento da disponibilidade de $P$, variou com a espécie do FMA. Esse comportamento também foi constatado neste trabal ho (Figura 1, aos 60 e 90 dias), o que reforça a hipótese de que a maior ou menor sensibilidade do fungo ao aumento da disponibilidade deP éum fator intrínseco à espécie, o que pode refletir na sua eficiência em estabelecer a simbiose com o hospedeiro (Abbott \& Robson, 1981; Lambais \& Cardoso, 1990).

\section{Massa do Material Seco da ParteAérea (MSPA)}

A produção de MSPA diferiu significativamente entre os tratamentos com fungos mi corrízicos apenas aos 60 e 90 dias (Quadro 1).

Aos 60 dias, os tratamentos com esem inoculação não diferiram estatisticamente entre si no tratamento sem adição de $\mathrm{P}$, no qual o teor de $\mathrm{P}$ disponível no substrato, determinado pelo método da resina, foi de $4 \mathrm{mg} \mathrm{kg}^{-1}$ (dados não apresentados). Esse teor, classificado como muito baixo (Raij et al., 1996), foi limitante mesmo na presença dos F MAs. $\mathrm{Na}$ dose de $25 \mathrm{mg} \mathrm{kg}^{-1}$ de $\mathrm{P}$ (15 $\mathrm{mg} \mathrm{kg}^{-1}$ de $\mathrm{P}$ disponível), a inoculação com $\mathrm{G}$. intraradices propiciou incremento de matéria seca 23,5 e 19,7\% superior à do tratamento com G. margarita e do controle, respectivamente. A percentagem de colonização radicular por $\mathrm{G}$. intraradices e $\mathrm{G}$. margarita era cerca de 45 e $10 \%$, respectivamente (Figura 1), permitindo que a primeira espécie fosse mais eficiente em auxiliar o crescimento do hospedeiro. Nas doses de 50 e $100 \mathrm{mg} \mathrm{kg}^{-1}$ de $\mathrm{P}$, a inoculação com $G$. margarita levou a produções de MSPA estatisticamente menores do que o controle. Nesse caso, G. margarita ainda estava em fase de estabelecimento da simbiose (crescimento lento), resultando numa depressão transitória de crescimento do hospedeiro, pois o FMA ainda não estaria contribuindo para o desenvolvimento da planta, mas constituindo um dreno de carbono. Estudos de Harris et al. (1985) revelaram que 17 e $8 \%$ dos fotoassimilados totais da planta de soja foram consumidos pelo fungo micorrízico aos 42 dias e no 
Quadro 1. Produção de matéria seca pela parte aérea de plantas de soja ( $\left(\mathrm{g} \mathrm{vaso}^{-1}\right)$ inoculadas com fungos micorrízicos arbusculares (G. margarita ou G. intraradices) ou não inoculadas (Controle), sob doses crescentes de fósforo, aos 15, 30, 60 e 90 dias do transplantio

\begin{tabular}{|c|c|c|c|c|c|}
\hline \multirow{2}{*}{ Fungo } & \multicolumn{5}{|c|}{ Dose de Fósforo (mg kg-1) } \\
\hline & $\mathbf{0}$ & 25 & 50 & 100 & 200 \\
\hline & \multicolumn{5}{|c|}{15 dias } \\
\hline Controle & $0,67 a$ & 0,78 a & $0,80 a$ & $0,90 \mathrm{a}$ & 0,85 a \\
\hline G. margarita & $0,73 \mathrm{a}$ & $0,85 a$ & $1,09 \mathrm{a}$ & $0,93 \mathrm{a}$ & $0,83 a$ \\
\hline \multirow[t]{2}{*}{ G. intraradices } & $0,67 a$ & $0,86 a$ & $1,11 \mathrm{a}$ & $0,75 a$ & $0,70 \mathrm{a}$ \\
\hline & \multicolumn{5}{|c|}{30 dias } \\
\hline Controle & $1,54 \mathrm{a}$ & $3,36 a$ & $4,40 \mathrm{a}$ & $5,64 a$ & 4,83 a \\
\hline G. margarita & $1,52 \mathrm{a}$ & 3,31 a & 3,88 a & $5,79 a$ & $4,64 \mathrm{a}$ \\
\hline \multirow[t]{2}{*}{ G. intraradices } & $1,67 \mathrm{a}$ & $3,57 a$ & $4,89 a$ & $4,68 \mathrm{a}$ & $5,43 a$ \\
\hline & \multicolumn{5}{|c|}{60 dias } \\
\hline Controle & $3,44 a$ & $18,42 \mathrm{~b}$ & $34,94 \mathrm{a}$ & $38,23 b$ & $36,68 \mathrm{ab}$ \\
\hline G. margarita & 3,66 a & $17,84 \mathrm{~b}$ & $28,08 \mathrm{~b}$ & $32,10 \mathrm{c}$ & 36,96 a \\
\hline \multirow[t]{2}{*}{ G. intraradices } & $4,68 \mathrm{a}$ & $22,04 a$ & 33,04 a & $42,26 a$ & $33,77 \mathrm{~b}$ \\
\hline & \multicolumn{5}{|c|}{90 dias } \\
\hline Controle & $3,80 \mathrm{~b}$ & $23,13 \mathrm{~b}$ & $48,90 \mathrm{~b}$ & $56,27 a$ & $49,60 \mathrm{a}$ \\
\hline G. margarita & $8,70 \mathrm{a}$ & $24,90 \mathrm{~b}$ & $47,25 \mathrm{~b}$ & $55,67 a$ & $52,20 a$ \\
\hline G. intraradices & $7,60 a$ & $30,80 a$ & $55,70 a$ & 55,95 a & 51,85 a \\
\hline
\end{tabular}

Médias seguidas pela mesma letra, nas colunas, dentro de cada época, não diferem entre si (teste t, 5\%).

final do ciclo da cultura, respectivamente. Conforme J akobsen (1995), pode-se observar depressão transitória de crescimento nas primeiras fases da colonização em plantas com reservas limitadas de carbono, principalmente em condições de alta disponibilidade de P. Bethlenfalvay et al. (1982b) também observaram inibição inicial de crescimento em soja, o que foi atribuído a uma demanda de carboidratos pelo endófito, numa fase em que a relação parte aérea/raiz ea capacidade fotossintética do hospedei ro eram baixas.

Aos 90 dias (Quadro 1), a inoculação com G. intraradices nos tratamentos com 0, 25 e $50 \mathrm{mg} \mathrm{kg}^{-1}$ deP acarretou maior acúmulo de MSPA. A inoculação com G. margarita somente resultou em diferença significativa superior à do controle no tratamento sem adição de $P$, o que demonstra que a simbiose com esse fungo foi menos eficiente em auxiliar o crescimento da planta, quando $P$ foi adicionado ao substrato. Nas doses de 100 e $200 \mathrm{mg} \mathrm{kg}^{-1}$ de $P$, não houve diferenças significativas entre os tratamentos com FMAs nem entre esses e o controle. Segundo J akobsen (1995), as diferenças na eficiência simbiótica entre fungos micorrízicos parecem estar ligadas muito mais à variação da capacidade de transporte de $\mathrm{P}$ pelo fungo do que às quantidades decarbono que esse consome do hospedeiro. Silveira \& Cardoso (1990) observaram que o FMA mais eficiente em promover o crescimento de feijoeiro foi aquel eque propiciou maior influxo deP pel osistema radicular da planta.

\section{Massa do Material Seco de Vagens (MSV)}

A análise estatística dos resultados da produção de vagens, obtidos aos 60 e90 dias, revelou interações duplas dos fatores épocas de colheita e fungos micorrízicos. Foram constatados (Quadro 2) efeitos positivos da inoculação com $\mathrm{G}$. intraradices apenas aos 90 dias, com incremento de produção de vagens em relação ao controle, que, por sua vez, não diferiu significativamente do tratamento inoculado com G. margarita. Novamente, foi constatada maior eficiência do primeiro fungo em relação ao segundo.

\section{Micélio Externo Ativo (MEA)}

Não houve efeito das doses de $P$ sobre o micélio externo ativo (MEA) produzi do nos tratamentos com FM As e controle, apenas aos 15 dias (Figura 2). Nas demais épocas, obtiveram-se ajustes de regressão significativos para os FMAs, com efeitos negativos das doses de P sobre o comprimento do MEA. No substrato do tratamento-controle, também foi observado MEA, resultado da presença de fungos não-micorrízicos, provavel mente introduzidos durante a instalação e realização do experimento. 
Quadro 2. Produção de matéria seca de vagens por plantas de soja $\left(g\right.$ vaso $\left.^{-1}\right)$ inoculadas com fungos micorrízicos arbusculares (G. margarita ou G. intraradices) ou não inoculadas (Controle), sob doses crescentes de fósforo, aos 60 e 90 dias do transplantio

\begin{tabular}{|c|c|c|c|c|c|c|}
\hline \multirow{2}{*}{ Fungo } & \multicolumn{5}{|c|}{ Dose de Fósforo $\left(\mathrm{mg} \mathrm{kg}^{-1}\right)$} & \multirow{2}{*}{ Média } \\
\hline & $\mathbf{0}$ & 25 & 50 & 100 & 200 & \\
\hline \multicolumn{7}{|c|}{60 dias } \\
\hline Controle & 0,13 & 0,66 & 0,97 & 0,98 & 1,00 & $0,75 a$ \\
\hline G. margarita & 0,20 & 0,69 & 1,48 & 0,75 & 1,46 & $0,92 \mathrm{a}$ \\
\hline G. intraradices & 0,18 & 0,86 & 1,23 & 0,78 & 0,87 & $0,78 \mathrm{a}$ \\
\hline \multicolumn{7}{|c|}{90 dias } \\
\hline Controle & 1,69 & 11,91 & 18,34 & 16,68 & 13,36 & $12,98 \mathrm{~b}$ \\
\hline G. margarita & 3,24 & 13,17 & 17,21 & 13,36 & 16,46 & $12,69 \mathrm{~b}$ \\
\hline G. intraradices & 3,94 & 15,52 & 19,60 & 17,16 & 19,13 & $15,07 \mathrm{a}$ \\
\hline
\end{tabular}

Médias de doses de fósforo seguidas pela mesma letra, dentro de cada época, não diferem entre si (teste t, 1\%).

As hifas de fungos não-micorrízicos nem sempre podem ser diferenciadas visualmente das hifas de FMAs, dificuldade que também foi relatada por Abbott \& Robson (1985) e Sylvia (1988).

Aos 30 e 60 dias, foram obtidos modelos matemáticos de regressão significativos para os tratamentos com FMAs, com altos valores de coeficiente de determinação, demonstrando influência negativa do aumento das doses de P sobre o MEA, de maneira análoga à col onização radicular (Figura 1). Notratamento-controle, oMEA somente foi influenciado pelas doses de $P$ aos 60 dias, o que reforça a afirmação de que o micélio originário de fungos não-micorrízicos estão menos sujeitos aos efeitos inibitórios das doses de P. Aos 90 dias, as produções de MEA nos tratamentos com G. margarita e no controle foram semelhantes, sem efeitos de doses de $P$. Esse comportamento pode explicar a menor eficiência deste fungo, quando comparado ao G. intraradices, o qual, mesmo prejudicado pelo aumento das doses deP, apresentou os maiores valores de MEA, o que provavelmente refletiu nas maiores produções de MSPA e MSV aos 90 dias (Quadros 1 e 2).

Embora G. margarita tenha atingido percentagem de col onização radicular superior à observada para G. intraradices aos 90 dias nas duas maiores doses de P (Figura 1), a produção de MEA por esse fungo foi inferior à produzida por G. intraradices em todas as doses de P (Figura 2). Aos 30 dias, o tratamento com $\mathrm{G}$. margarita apresentou mai or desenvolvimento inicial deMEA notratamento sem adição deP; contudo, provavel mente, em virtude da fal ta de adaptação ao substrato (Abbot $\&$ Robson, 1985), passou a apresentar menores produções de MEA que o tratamento com $\mathrm{G}$. intraradices nas

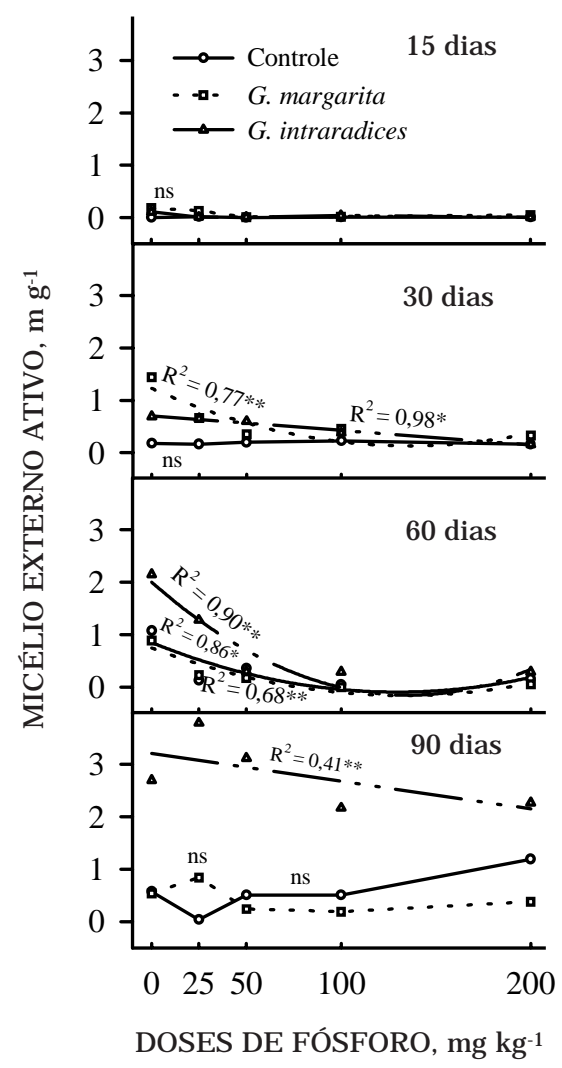

Figura 2. Estimativa do comprimento de micélio externo ativo em substrato cultivado com soja inoculada com fungos micorrízicos arbusculares (G. margarita ou G. intraradices) ou não inoculada (Controle), aos 15, 30, 60 e 90 dias do transplantio, considerando as doses de $\mathbf{P}$. * Modelos matemáticos de regressão significativos a $5 \%$; $* *$ = significativos a $1 \%$; ns = nãosignificativos pelo teste $F$. 
épocas seguintes, com valores estatisticamente iguais aos do controle. A concentração de $P$ no hospedeiro parece influenciar a quantidade ou a composição dos exsudatos radiculares, o que pode afetar o crescimento do micélio externo dos FMAs (Tawaraya et al., 1996). Fatores físicos, químicos e biológicos do substrato também podem afetar a integridade do micélio externo e comprometer a transl ocação de nutrientes dos F MAs ao hospedeiro (Sylvia, 1988).

O comprimento de MEA de G. intraradices aumentou com o tempo em todas as doses de $P$ (Figura 2), enquanto no tratamento com G. margarita foi semelhante ao do controle. Schubert et al. (1987), Sylvia (1988) e Hamel et al.(1990) observaram que, em geral, a percentagem de MEA em relação ao mi célio externo total (MET) é alta no início do estabelecimento da simbiose, diminuindo conforme sua evolução. Neste estudo, porém, observou-se o contrário, principalmente para G. intraradices, para o qual a proporção de MEA em relação ao MET foi cerca de $5 \%$ aos 15 dias, chegando a cerca de $20 \%$ aos 90 dias (dados não apresentados).

Como a absorção de nutrientes pela hifa do fungo ocorre por processo ativo, o aumento do MEA, produzido por G. intraradices e, conseqüentemente, da superfície disponível para absorção, melhoraria o estado nutricional do hospedeiro associado a esse fungo, principalmente nas doses mais baixas de $P$. Entretanto, a planta teve pouco tempo para se beneficiar da interação, pois já havia concluído mais de $75 \%$ do seu ciclo aos 90 dias.

\section{Micélio Externo Total (MET)}

Embora alguns autores (Sylvia, 1988; Hamel et al., 1990) tenham sugerido a subtração da quantidade de micélio externo total (MET) encontrada nos substratos-controles daquela dos substratos inoculados com F MAs, neste estudo, esse procedimento não foi realizado. Em algumas situações, houve mai or comprimento de MET no tratamento-controle, supostamente pelos mesmos motivos apresentados para MEA, além de que interações não conhecidas podem ocorrer entre os FMAs efungos não-micorrízicos. Novamente, houve dificuldade de diferenciação visual entreas hifas de FMAs e os fungos não-micorrízicos (Abbott \& Robson, 1985; Sylvia 1988).

De maneira geral, houve tendência de aumento da produção de MET ao longo do tempo (o tratamento com $\mathrm{G}$. intraradices produziu, na média das doses deP , 3,4 $\mathrm{m} \mathrm{g}^{-1}$, aos 15 dias, e 7,5 $\mathrm{m} \mathrm{g}^{-1}$, aos 90 dias), tendendo o aumento das doses de $\mathrm{P}$ a diminuir o MET (Figura 3), embora não se tenham obtidos ajustes de model os matemáticos significativos. Resultados com a mesma tendência foram obtidos por Miranda \& Harris (1994).

É interessante notar que apenas aos 60 dias ficou bem evidente a diminuição do MET com o aumento das doses de P. Esta observação ressalta a importância deavaliar a produção de MET, bem como as outras variáveis relacionadas em diversas fases do cicl o do hospedeiro (Bethlenfalvay et al., 1982d), visto que o estabel ecimento da simbiose com o FMA é um processo dinâmico, em que as mais variadas condições do hospedeiro influenciarão o simbionte, e vice-versa, tanto no quese refereà disponibilidade de $\mathrm{P}$ quanto à adaptação do FMA às condições do meio.

Alguns autores (Abbott \& Robson, 1985; Sylvia, 1988; Hamel et al., 1990) levantaram questão sobre qual a longevidade da hifa no solo, pois observaram grandeincremento da col onização radicular, mas não foi constatado aumento do mi cél io externo no mesmo período. Uma explicação para o fato seria a rápida reciclagem da hifa externa ou sua degradação por microrganismos do solo. Nesse experimento, houve, em geral, aumentos do MET com o tempo (Figura 3).

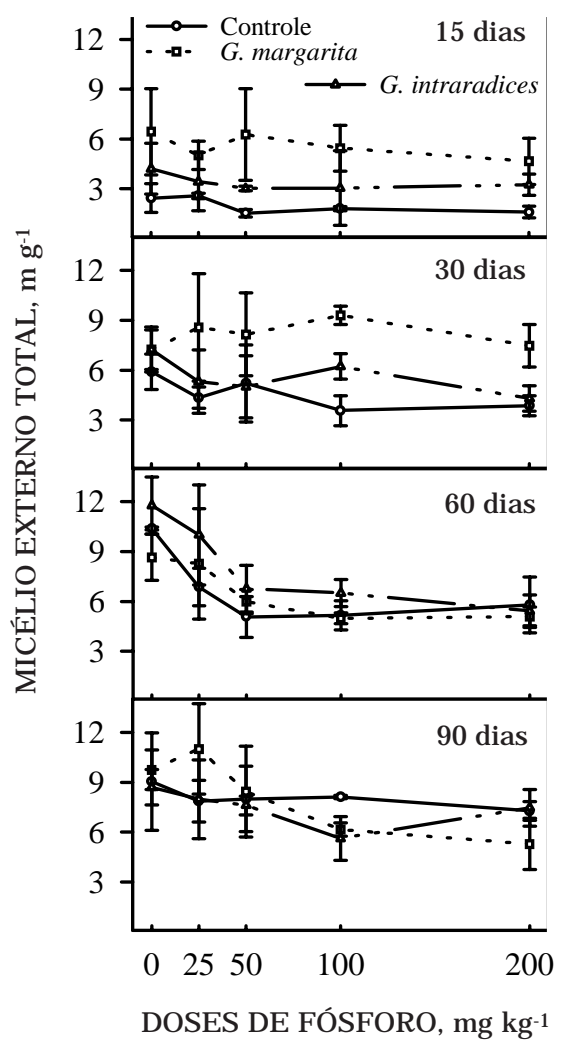

Figura 3. Estimativa do comprimento de micélio externo total em substrato cultivado com soja inoculada com fungos micorrízicos arbusculares (G. margarita ou G. intraradices) ou não inoculada (Controle), aos 15, 30, 60 e 90 dias do transplantio, considerando as doses de $\mathbf{P}$. Barras verticais representam o desvio-padrão de três repetições, quando maiores que os símbolos. 
Segundo Abbott \& Robson (1985), a capacidade de o FMA col onizar o hospedeiro e produzir mi célio externo deve ser considerada na seleção de fungos mais eficientes. Por outrolado, Schubert et al . (1987) salientaram que há pouca relação entre MET e absorção de nutrientes, motivo pel o qual se deve dar mais importância à distribuição espacial dos dois componentes do micélio no solo, considerando também a parte ativa. Sob esse aspecto e nas condições deste experimento, G. margarita foi o fungo menos eficiente para as plantas de soja, por ter produzido menos MEA (Figura 2), embora apresentasse nas duas primeiras épocas, tendência de maior produção de MET (Figura 3). Essa baixa eficiência de G. margarita em soja foi observada anteriormente, mas apenas para aspectos relacionados com a planta, ou seja, produção de biomassa (Cardoso, 1986; Lambais \& Cardoso, 1990). Neste experimento, os aspectos relacionados com o FMA (produção de MEA e MET) permitiram sugerir que a menor eficiência de G. margarita em auxiliar o crescimento da soja pode ser atribuída, dentre outros fatores, à menor produção de micélio externo ativo.

\section{Nutrientes}

Com relação à concentração de nutrientes na parte vegetativa (fol has + pecíol os + ramos), não se observaram efeitos expressivos da presença dos FMAs, inclusive para o fósforo (dados não apresentados). Contudo, a concentração de ferro e manganês nas vagens foi influenciada pela presença dos FMAs, dependendo da dose de P (Figuras 4a e4b, respectivamente). A disponibilidade inicial de $\mathrm{Fe}$ e $\mathrm{Mn}$ no substrato (93 e $11 \mathrm{mg} \mathrm{kg}^{-1}$, respectivamente) é considerada alta (Raij et al., 1996). Entretanto, a inoculação com FMAs nos tratamentos com as menores doses de $\mathrm{P}$ resultou em menores concentrações de Fee $\mathrm{Mn}$ nas vagens. É possível que esse comportamento esteja relacionado com a maior percentagem de col onização radicular, comprimento de MEA e MET obtidos nesses tratamentos (Figuras 1, 2 e 3, respectivamente). Com o aumento das doses de $P$, com a redução da col onização, MEA e MET, as concentrações de Fe e Mn aumentaram atéà dose de $100 \mathrm{mg} \mathrm{kg}^{-1}$, com posterior diminuição na dose $200 \mathrm{mg} \mathrm{kg}^{-1}$, com um comportamento quadrático. Nas menores doses de P, o controle sem FMA apresentou as maiores concentrações de Fe e $\mathrm{Mn}$, com dimi nuiçãolinear com o aumento das doses. Como na dose de $200 \mathrm{mg} \mathrm{kg}^{-1}$ de $\mathrm{P}$, o decréscimo da concentração de Fe e $\mathrm{Mn}$ nas vagens ocorreu tanto na presença quanto na ausência dos F MAs, parece haver um efeito atribuível ao $P$, provavel mente pela formação de complexos de baixa solubilidade com o Fe e $\mathrm{Mn}$, tornando-os menos disponíveis (Habte \& Soedarjo, 1996). Esses resultados revelam o efeito protetor dos FMAs em condições de alta disponibilidade de Fe e Mn, quando a col onização radicular eo micélio externo não são limitados pela disponibilidade deP.

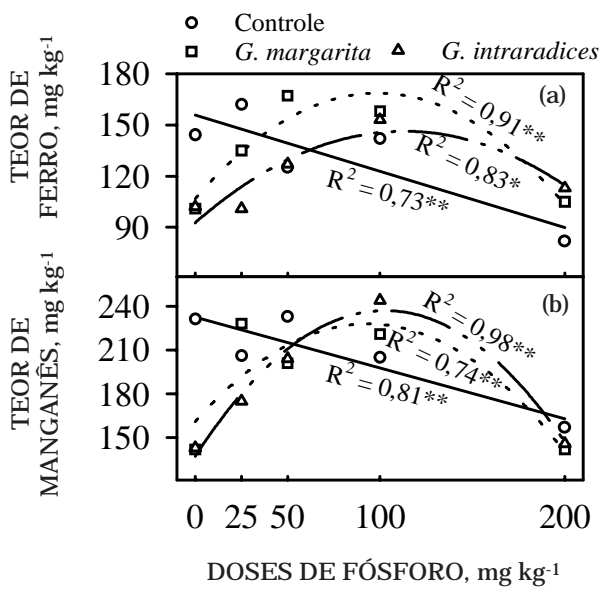

Figura 4. Teores de Fe (a) e Mn (b) nas vagens de plantas de soja inoculadas com fungos micorrízicos arbusculares (G. margarita ou G. intraradices) ou não inoculadas (Controle), aos 90 dias do transplantio, considerando as doses de P.* Modelos matemáticos de regressão significativos a $5 \%$; ** =significativos a $1 \%$ pelo teste $F$.

Cardoso (1985; 1986) também observou diminuições da concentração de $\mathrm{Mn}$ em tecidos de soja na presença de FMAs. Pacovsky et al. (1986) constataram, além de menores teores de $\mathrm{Mn}$, diminuições nos teores deF eem plantas mi corrizadas, sugerindo que mudanças de $\mathrm{pH}$ na micorrizosfera podem alterar o estado de oxidação do $\mathrm{Mn}$, o que levaria à alteração da sua absorção. Contudo, os mecanismos pelos quais a micorrização auxilia a proteção da planta contra alta disponibilidade de Fe e Mn, ainda não são conhecidos, sendo necessários mais estudos para sua elucidação.

\section{CONCLUSÕES}

1. O fungo micorrízico arbuscular G. margarita apresentou menor eficiência no estabel ecimento da simbiosecom soja, com fase de crescimentolento mais prol ongada emenor produção de micélio externoativo.

2. O incremento das doses de fósforo afetou, de forma diferenciada, o estabelecimento da simbiose entre cada um dos fungos mi corrízicos ar busculares ea soja.

3. O incremento das doses de $P$ causou decréscimo do micélio externo ativo, mas não influenciou o micélio externo total na mesma intensidade.

4. Quando ocorreu alta disponibilidade de Fe e Mn no substrato, a presença dos fungos mi corrízicos arbusculares auxiliou o hospedeiro na prevenção da absorção excessiva desses íons, quando suficiente colonização radicular foi atingida. 


\section{AGRADECIMENTOS}

AoProf. Dr. Raymond S. Pacovsky, pelas sugestões apresentadas e pela correção do Summary; aos técnicos Denise Lourdes Colombo Mescolotti e L uís Fernando Baldesin, do Laboratório de Microbiologia do Solo (Departamento de Solos e Nutrição de Plantas da ESALQ/USP), pelos auxílios prestados durante a instalação e realização do experimento.

\section{LITERATURA CITADA}

ABBOTT, L.K. \& ROBSON, A.D. Infectivity and effectiveness of vesicular arbuscular mycorrhizal fungi: effect of inoculation type. Aust. J . Agric. Res., 32:631-639, 1981.

ABBOTT, L.K. \& ROBSON, A.D. Formation of external hyphae in soil by four species of vesicular-arbuscular mycorrhizal fungi. New Phytol., 99:245-255, 1985.

BETHLENFALVAY, G.J .; BROWN, M.S. \& PACOVSKY, R.S. Parasitic and mutualistic associations between a mycorrhizal fungus and soybean: development of the endophyte. Phytopathology, 72:894-897, 1982a.

BETHLENFALVAY, G.J .; BROWN, M.S. \& PACOVSKY, R.S. Parasitic and mutualistic associations between a mycorrhizal fungus and soybean: development of the host plant. Phytopathology, 72:889-893, 1982b.

BETHLENFALVAY, G.J .; BROWN, M.S. \& PACOVSKY, R.S. Relationships between host and endophyte development in mycorrhizal soybeans. New Phytol. 90:537-543, 1982c.

BETHLENFALVAY, G.J .; PACOVSKY, R.S.; BROWN, M.S. \& FULLER, G. Mycotrophic growth and mutualistic development of host plant and fungal endophyte in an endomycorrhizal symbiosis. Plant Soil, 68:43-54, 1982d.

CARDOSO, E.J.B.N.; ANTUNES, V.; SILVEIRA, A.P.D. \& OLIVEIRA, M.H.A. Eficiência de fungos micorrízicos vesículo-arbusculares em porta-enxertos de citrus. R. Bras. Ci. Solo, 10:25-30, 1986.

CARDOSO, E.J .B.N. Efeito de micorriza vesículo-arbuscular e fosfato-de-rocha na simbiose soja-Rhizobium. R. Bras. Ci. Solo, 9:125-130, 1985.

CARDOSO, E.J .B.N. Eficiência de fungos micorrízicos vesículoarbusculares em soja, com Rhizobium japonicum e fosfato de rocha, em função do tipo de sol o. R. Bras. Ci. Solo, 10:1723, 1986.

CARDOSO-FILHO, J .A.; PACOVSKI, R.S. \& CARDOSO, E.J .B.N. Growth and metabolic activity of the extramatrical mycelium of endomycorrhizal maize plants. R. Bras. Ci. Solo, 23:807-815, 1999.

FAQUIN, V. Cinética da absorção de fosfato, nutrição mineral, crescimento e produção da soja sob influência de micorriza vesículo-arbuscular (MVA). Piracicaba, Escola Superior de Agricultura "Luiz de Queiroz", Universidade de São Paulo, 1988. 136p. (Tese de Doutorado)
GERDEMANN, J.W. \& NICOLSON, T.H. Spores of mycorrhyzal endogoneextracted from soil by wet sieving end decanting. Trans. Br. Mycol. Soc., 46:235-244, 1963.

GIOVANNETI, M. \& MOSSE, B. An evaluation of techniques for measuring vesicular arbuscular mycorrhizal infection in roots. New Phytol., 84:489-500, 1980.

HABTE, M. \& SOEDARJ O, M. Response of Acacia mangium to vesicular-arbuscular mycorrhizal inoculation, soil $\mathrm{pH}$, and soil P concentration in an oxisol. Can. J. Bot., 74:155-161, 1996.

HAMEL, C.; FYLES, H. \& SMITH, D.L. Measurement of development of endomycorrhizal mycelium using three different vital stains. New Phytol., 115:297-302, 1990.

HARRIS, D.; PACOVSKY, R.S. \& PAUL; E.A. Carbon economy of soybean-Rhizobium-Glomus associations. New Phytol., 101:427-440, 1985.

J AKOBSEN, I. Transport of phosphorus and carbon in VA mycorrhizas. In: VARMA, A. \& HOCK, B., eds. Mycorrhiza: structure, function, molecular biology and biotechnology. Berlin, Springer-Verlag, 1995. p.297-324.

LAMBAIS, M.R. \& CARDOSO, E.J .B.N. Response of Styl osanthes guianensis to endomycorrhizal fungi inoculation as affected by lime and phosphorus applications. Plant Soil, 129:283289, 1990.

MELLONI, R. \& CARDOSO, E.J .B.N. Quantificação de micélio extrarradicular de fungos micorrízicos arbusculares em plantas cítricas. I. Método empregado. R. Bras. Ci. Solo, 23:53-58, 1999a.

MELLONI, R. \& CARDOSO, E.J.B.N. Quantificação de micélio extrarradicular de fungos micorrízicos arbusculares em plantas cítricas. II. Comparação entre diferentes espécies cítricas e endófitos. R. Bras. Ci. Solo, 23:59-67, 1999b.

MINHONI, M.T.A.; CARDOSO, E.J .B.N. \& EIRA, A.F. Efeitos da adição de fosfato de rocha, bagaço de cana-de-açúcar, fosfato solúvel e fungo micorrízico no crescimento e na absorção de nutrientes por plantas de soja. R. Bras. Ci. Solo, 17:173178, 1993.

MIRANDA, J.C.C. \& HARRIS, P.J . The effect of soil phosphorus on the external mycelium growth of arbuscular mycorrhizal fungi during the early stages of mycorrhiza formation. Plant Soil, 166:271-280, 1994.

NAGAHASHI, G.; DOUDS J r., D.D. \& ABNEY, G.D. Phosphorus amendment inhibits hyphal branching of the VAM fungus Gigaspora margarita directly and indirectly through its effect on root exudation. Mycorrhiza, 6:403-408, 1996.

OJ ALA, J.C.; J ARRELL, W.M.; MENGE, J .A. \& J OHNSON, E.L.V. Comparison of soil phosphorus extractants as predictors of mycorrhizal dependency. Soil Sci. Soc. Am. J ., 47:958-962, 1983.

PACOVSKY, R.S.; BETHLENFALVAY, G.J . \& PAUL, E.A. Comparison between P-fertilized and mycorrhizal plants. Crop Sci., 26:151-156, 1986.

PHILLIPS, J .M. \& HAYMAN, D.S. Improved procedures for clearing roots and staining parasitic and vesiculararbuscular mycorrhizal fungi for rapid assesment of infection. Trans. Br. Mycol. Soc., 55:158-161, 1970. 
RAIJ , B. van; CANTARELLA, H.; QUAGGIO, J .A. \& FURLANI, A.M.C. Recomendações de adubação e calagem para o estado deSão Paulo. Campinas, Instituto Agronômico deCampinas, 1996. 285p. (Boletim técnico, 100)

SARRUGE, J R. \& HAAG, H.P. Análises químicas em plantas. Piracicaba, Escola Superior de Agricultura Luiz deQueiroz, 1974. 56p.

SCHUBERT, A.; MARZACHÍ, C.; MAZZITELLI, M.; CRAVERO, M.C. \& BONFANTE-FASOLO, P. Development of total and viable extraradical mycelium in the vesicular-arbuscular mycorrhizal fungus Glomus clarum Nicol \& Schenck. New Phytol., 107:183-190, 1987.
SILVEIRA, A.P.D. \& CARDOSO, E.J .B.N. Kinetics of phosphorus uptake, growth and mineral nutrition of mycorrhizal and non-mycorrhizal bean (Phaseol us vulgaris L.). In: NORTH AMERICAN CONFERENCE ON MYCORRHIZAE, 8., Wyoming, 1990. Abstracts. Wyoming, J ackson, 1990. p.264.

SIQUEIRA, J .O.; HUBBELL, D.H. \& VALLE, R.R. Effects of P on formation of the VAM symbiosis. Pesq. Agropec. Bras., 19:1465-1474, 1984.

SYLVIA, D.M. Activity of external hyphae of vesicular-arbuscular mycorrhizal fungi. Soil Biol. Biochem., 20:39-43, 1988.

TAWARAYA, K.; WATANABE, S. \& YOSHIDA, E. Effect of onion (Allum cepa) root exudates on the hyphal growth of Gigaspora margarita. Mycorrhiza, 6:57-59, 1996. 